\title{
CRITERIA AND PROCEDURES FOR ASSESSING OCCUPIED VOLUME INTEGRITY
}

\author{
Michael Carolan \\ A. Benjamin Perlman \\ David C. Tyrell \\ Volpe National Transportation Systems Center \\ US Department of Transportation \\ Cambridge, MA 02142
}

\begin{abstract}
With the potential for tremendous growth in the passenger rail industry, providing for the safety of the train-riding public and the crews who transport them becomes an ever-greater priority. To provide for safety while making best use of its resources and to facilitate passenger rail industry growth, the Federal Railroad Administration (FRA), in consultation with the rail industry, has developed alternative Criteria and Procedures for assessing the crashworthiness and occupant protection measures of rail passenger equipment. These Criteria and Procedures are intended to be applicable to a wide range of equipment designs, particularly equipment designs not complying with current U.S. standards and regulations. Because the latest technology in rail equipment crashworthiness has been used to develop the Criteria and Procedures, aspects of the resulting Criteria and Procedures are fundamentally different from their corresponding regulations. While technical results from sophisticated analyses and tests have been necessary, judgment was also needed to develop the Criteria and Procedures. This judgment was provided by the Engineering Task Force (ETF), and ultimately accepted by FRA. The ETF is a government/industry working group, organized under the auspices of the Railroad Safety Advisory Committee (RSAC).
\end{abstract}

The Criteria and Procedures are intended to provide an engineering-based methodology for comparing the crashworthiness of alternatively-designed equipment with that of compliant designs. One particularly important aspect of passenger car crashworthiness is occupied volume integrity (OVI). It is essential that all passenger vehicles meet some base minimum level of OVI. A primary goal of crashworthiness is to maintain a volume for occupants to ride out a collision. In the U.S., this base level has been demonstrated through a vehicle's ability to react a quasi-static load of 800,000 pounds along its line of draft without experiencing permanent deformation. This car-level requirement has existed, in some form, since the early $20^{\text {th }}$ century. However, alternatively-designed vehicles may not be able to demonstrate the ability to support this load, but may still prove to be equivalently crashworthy. Based on analyses performed on conventional and alternatively-designed passenger equipment, three options have been developed to demonstrate the OVI of alternatively-designed equipment. These options consist of three load magnitudes placed along the collision load path with a corresponding pass/fail criterion for each load. OVI may be demonstrated by sustaining an 800,000 pound load with no permanent deformation, a $1,000,000$ pound load with limited permanent deformation, or a $1,200,000$ pound load without exceeding the crippling load of the occupied volume.

This paper discusses the pass/fail criteria associated with each option, the analysis and test procedures used in applying each option, and the technical basis used in developing the Criteria and Procedures for OVI evaluation. By applying such techniques, the results of evaluations of alternatively-designed equipment can be compared with the Criteria values for compliant designs. In this manner, the crashworthiness performance of alternatively-designed equipment can be assessed relative to the performance of compliant designs. A companion paper to this one discusses the development of the train-level Criteria and Procedures.

This material is declared a work of the U.S. Government and is not subject to copyright protection in the United States.

Approved for public release. Distribution is unlimited. 


\section{BACKGROUND}

FRA, with the assistance of the Engineering Task Force (ETF), has developed alternative Criteria and Procedures for assessing the crashworthiness and occupant protection features of rail passenger equipment that does not comply with the existing regulations. The Criteria are both the conditions to be evaluated and the metrics for assessment. For the existing 800,000 pound compressive "buff strength" requirement, the Criteria are an 800,000 pound load and no permanent deformation within the car body. The Procedures are the testing and/or analysis methods employed in evaluating the structure's ability to meet the Criteria. For the existing 800,000 pound compressive strength test, the procedures are a quasistatic compression test of a full carbody structure.

\section{Mission}

The ETF reported to the Passenger Safety Working Group of the Railroad Safety Advisory Committee (RSAC). The mission of the Task Force was to produce a set of technical Criteria and Procedures for evaluating passenger rail equipment built to alternative designs. The technical evaluation Criteria and Procedures provide a means of establishing whether equipment of an alternative design result in at least equivalent performance to that of equipment designed in accordance with the structural standards in the Passenger Equipment Safety Standards (49 CFR Part 238). The initial focus of this effort was on Tier I crashworthiness and occupant protection standards. The product of this effort is a report, entitled "Technical Criteria and Procedures for Evaluating the Crashworthiness and Occupant Protection Performance of Alternatively-Designed Passenger Rail Equipment for Use in Tier I Service” [1].

\section{Goals}

The Task Force set out to meet the following goals:

- Utilize the collective "best" thinking in the passenger rail industry;

- Produce clear, realistic technical Criteria and Procedures for demonstrating equivalent performance;

- Define the analysis and testing necessary to demonstrate the integrity of any specific design;

- Provide clear pass/fail analysis and testing criteria; and

- Work expeditiously so that the technical Criteria and Procedures are available to sponsors of potential passenger rail service.

It was not intended that the Task Force attempt to identify every possible means of determining the performance of alternative designs, nor did FRA anticipate that the availability of technical Criteria and Procedures would eliminate the need to apply sound engineering judgment in reviewing requests for waivers and other approvals. However, it was anticipated that the availability of technical Criteria and Procedures could substantially reduce the uncertainty associated with demonstrating equivalent safety or alternative compliance.

\section{Occupied Volume Integrity}

The conventional approach to passenger vehicle crashworthiness applied in the United States has been to require that the underframe of the car be capable of maintaining its integrity when subjected to a large compressive load at the coupler locations at either end of the car. The present strength requirement is for a car to remain elastic when subjected to 800,000 pounds of force loaded along the line of draft (the imaginary line running from the coupler at one end of the car to the other). This load is shown schematically in Figure 1.

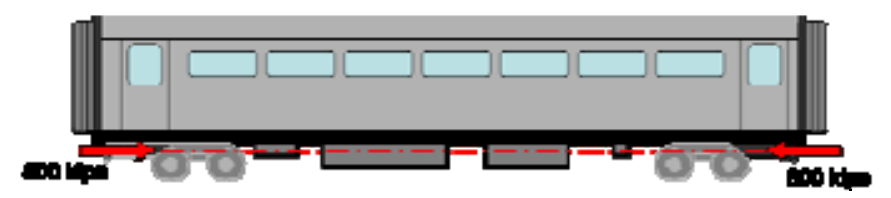

Figure 1. 800,000 Pounds on Line of Draft

The practice of applying a large compressive load to the underframe of the car as a measure of occupant protection stretches back to the early $20^{\text {th }}$ century. At that time, the United States Post Office had in service a number of Railway Post Office (RPO) Cars. The original RPO cars were baggage cars that had tables, chairs, and lighting installed so that postal clerks could sort mail while a train was en route. Unfortunately, in many railroad accidents of the day these baggage cars offered little protection to the clerks inside, resulting in serious injuries and fatalities. As a means of increasing occupant protection, the Railway Mail Service (RMS) Specification was published in 1912. One requirement in this specification was for RPO cars to be capable of resisting 400,000 pounds applied compressively along the line of draft without experiencing permanent deformation. In future versions of this specification, a factor of safety of 2 was included, bringing the effective load up to 800,000 pounds [2].

In response to a number of fatal accidents involving compromised occupied volumes, the Association of American Railroads (AAR) issued a Recommended Practice in 1939 to address car body structure. This Recommended Practice adopted a number of requirements of the RMS Specification, including the compressive strength of the car body. In 1945 this recommendation was adopted into Standard S-034, "Specifications for the Construction of New Passenger Equipment Cars." Federal law has applied this requirement to all MU locomotives built new after April 1, 1956, and operated in trains having a total empty weight of 600,000 pounds or more. See 49 CFR 229.141(a)(1). It was not until 1999, however, that 49 CFR 238.203 expanded this 800,000-pound 
static strength requirement as a federal regulation applicable to all intercity passenger and commuter rail equipment.

This line of draft strength requirement has remained the cornerstone of OVI evaluation for nearly a century, for a number of reasons. The pass/fail criterion of no permanent deformation anywhere in the car is straightforward to implement and can be readily examined visually and measured with strain gages. If the test is conducted properly and successfully, the vehicle remains in its original condition and can therefore enter service following the test. The nondestructive nature of the test makes it an economical test to perform as the first manufactured vehicle serves both as test article and proven, deliverable product.

Additionally, the proof strength approach to crashworthiness provides additional crashworthiness benefits. While the original intent of this approach was to maintain some level of protection from loss of occupied volume, this requirement has increased in its importance as other crashworthiness features have been incorporated within the car. For example, standards and regulations also specify the minimum strength of the corner and collision posts on a passenger vehicle. For an end frame to be successful in preventing intrusion from impacts above the floor, the structure supporting the end frame must itself be sufficiently strong. A strong end frame that is at the end of a weak occupied volume may prevent intrusion at the end of the car but cause loss of occupied volume elsewhere in the vehicle as collision loads travel through the occupied volume.

\section{TECHNICAL BASIS FOR CRITERIA}

The objective of this effort was to develop Criteria and Procedures for assessing the crashworthiness and occupant protection performance of alternatively-designed equipment to be used in Tier I service. This section describes the technical basis for how the selected criteria provide a comparable level of crashworthiness to the existing regulation on OVI.

Because the OVI Criteria have been adjusted from the criteria within the regulation, an additional evaluation has been added to the overall framework of the Criteria and Procedures. A train-level impact scenario has been added as a second evaluation of a vehicle's ability to maintain sufficient space for occupants to "ride out" a collision. The static testing and analysis described in this paper works together with the scenario described in the companion paper [5] to establish the crashworthiness of the vehicle undergoing evaluation.

Preservation of occupied volume is an essential part of the crashworthiness of any rail car. Conventional passenger railcars are designed to carry their service loads along the line of draft. Because longitudinal collision loads are also introduced along the line of draft in conventional passenger cars, the regulations require a minimum elastic resistance to a load along that load path. The load specified in the regulations is readily applied to cars with a traditional buff stop arrangement and an apparent line of draft. However, application of this load presents some difficulty for vehicles without conventional buff stops, a difficult-to-define line of draft, or a collision load path that differs from the service load path.

Preserving occupied volume is accomplished primarily with strength of the structure. If the occupied compartment is sufficiently strong, there will be sufficient, survivable space for the occupants. Allowing portions of the vehicle to crush in a predetermined manner can limit the forces applied to the structure surrounding the occupied volume and control the decelerations of the cars. Conventional practice is oriented toward making the individual cars uniformly strong, and principally attempts to control the behavior of individual cars during a collision. The Crash Energy Management (CEM) approach is train-oriented, controlling the load into the occupied volume and apportioning the structural crushing to unoccupied areas throughout the train. This approach is illustrated schematically in Figure 2.

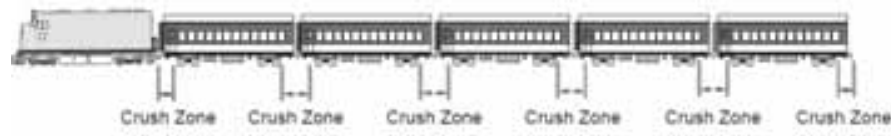

Figure 2. Schematic Illustration of Crush Zone Locations in Commuter Rail Passenger Train

A strength-based approach was used in developing the Options in the OVI Criteria for alternatively-designed equipment. Carlevel OVI requirements are designed to work in concert with the train-level scenario to assure that the space for the passengers and crew is preserved in moderately severe accident conditions. Additionally, as two of the Options allow permanent deformation to occur, it is anticipated that analysis will be used to demonstrate that a car meets a particular Option. Analysis requires proper validation through nondestructive testing of the vehicle undergoing evaluation. Testing and analysis procedures are discussed in detail in the Procedures section of this paper.

One of the difficulties in evaluating alternatively-designed equipment to the requirements of the Federal Regulations involves the requirements for particular design features. For example, evaluating a vehicle's compliance with 49 CFR 238.203 requires a design capable of supporting 800,000 pounds along the line of draft, whether or not it is equipped with CEM features. Other areas of the CFR require specific structures for collision posts (§238.211) and corner posts (§238.213). This design-based requirement may be difficult to apply to a vehicle with a corner structure that is intended to function in the same way as a corner post, but is not, in fact, a post. 
The U.S. Regulations are readily applied to a vehicle that has been designed with the specific structures in the specific locations that the requirements will be evaluating. However, it is possible to develop a design that provides an equivalent level of passenger and crew safety to conventionally-designed equipment without using the specific structures in the specific locations specified in the existing Regulations. As part of the approach undertaken by the ETF, performance Criteria that are "design neutral" were developed for a number of areas of safety which have frequently been the subject of waiver requests by parties wishing to operate vehicles of an alternative design.

In recognizing the variety of designs currently operating in other parts of the world (e.g. high-floor, partial-low floor, coupled, articulated, EMU, DMU, multi-level, etc.), the OVI Criteria Options were designed to be readily applied to a variety of designs. Rather than placing the load along the line of draft, the Options all place the load along the collision load path. This load placement ensures that the OVI is evaluated in a manner based on the way it will be loaded during a collision. The Options were developed to help ensure a comparable level of OVI among vehicles meeting any one of the Options. A design needs to demonstrate that it meets at least one of the Options, or it otherwise must comply with the regulation itself.

During the ETF process for developing load magnitudes and pass/fail criteria for OVI, it was decided that having multiple options would allow a variety of design methodologies to be evaluated while still establishing a baseline level of safety. The resulting Criteria consist of three Options, any one of which may be chosen in evaluating a particular design.

Option A requires a car body to support a quasi-static load applied along the collision load path without permanent deformation of the body structure. This option is most closely related to the U.S. requirement of 800,000 pounds applied along the line of draft. The key difference in procedure is the location where loads are applied.

In a conventionally-designed car, the service loads and the collision loads are both transmitted along the line of draft. For alternatively-designed equipment, the vehicle may feature one load path for service loads, but a different load path for collision loads. Since the occupied volume is subject to much more severe loading during collisions than normal service, it was determined that the evaluation load should be placed along the collision load path, even if that is different from the service load path.

The locations within the collision load path where high longitudinal loads can be applied to the car body structure are determined as part of the design development. As the location(s) where high collision loads enter the occupied volume may be inaccessible for testing, detailed analysis of the carbody is one procedure for demonstrating a design meets Option A.

Option B requires a car body to support a quasi-static load applied along the collision load path with a limited amount of permanent deformation. The target load value would be greater than the 800,000 pounds required by the current regulation. Because the load magnitude would be increased, the pass/fail criterion allows some permanent deformation under this loading condition. Values chosen for "limited permanent deformation" were developed based on finite element analyses performed on conventional and alternatively-designed passenger equipment under the given load.

Option $\mathrm{C}$ requires a car body to support a large quasi-static load along the collision load path without crippling the body structure. Crippling of the body structure has been defined as the largest load the occupied volume can support. This value is indicated by the peak on a load-displacement characteristic. An example load-displacement characteristic, with crippling load indicated, is shown in Figure 3.

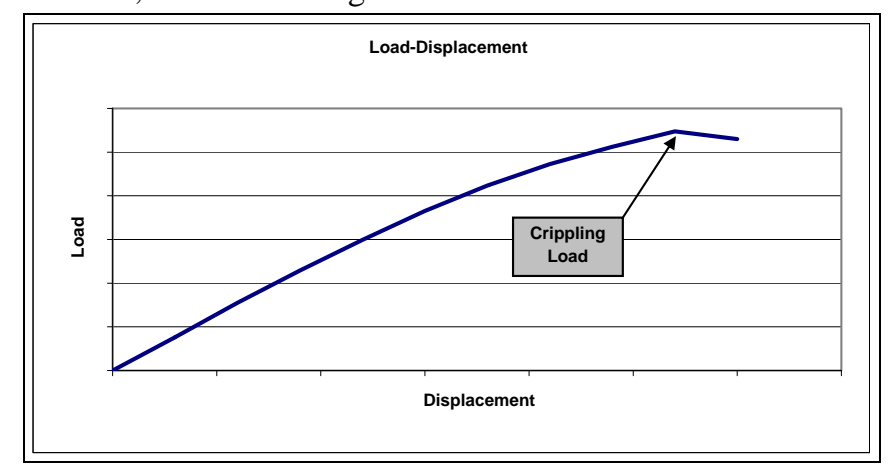

Figure 3. Example Load-displacement Characteristic Indicating Crippling Load

A large load value for the criteria is appropriate to provide a safety margin over the minimum elastic load met by Tier Icompliant equipment. The load magnitude was chosen based on analysis of the capabilities of conventional and alternatively-designed equipment. Under the definition of "crippling load" used in the Criteria and Procedures Report, localized buckling is not considered crippling of the occupied volume. Rather, the body structure is considered crippled when a global maximum load has been reached during quasi-static analysis.

Figure 4 shows a typical load-displacement characteristic from a finite element analysis of the occupied volume. In this particular analysis, the load features a local maximum followed by a global maximum. This local maximum corresponds to a localized buckling in the roof structure of the car. However, this localized buckling does not prevent the occupied volume 
from bearing more load. The second peak occurs when the underframe buckles. At this point, the occupied volume has been crippled, as with increasing displacement the occupied volume cannot sustain increasing load.

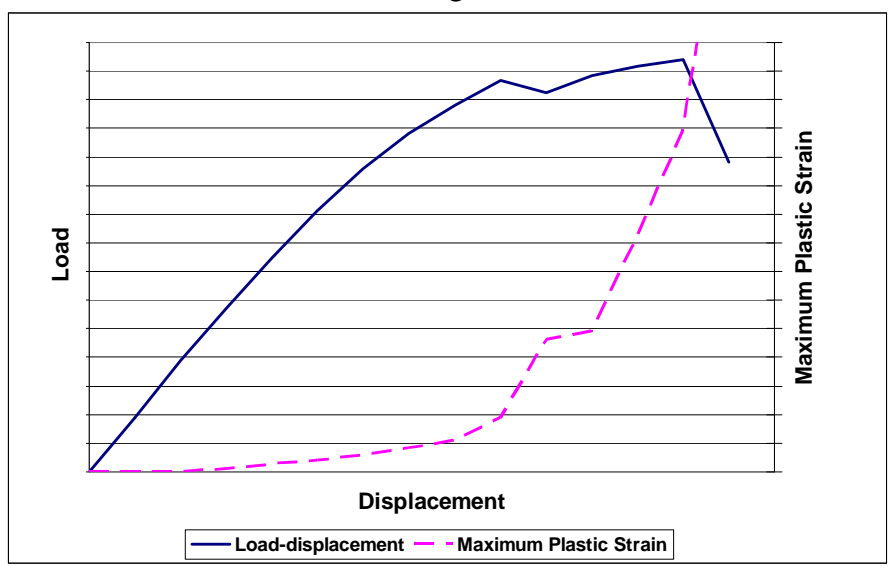

Figure 4. Typical Load-displacement and Plastic Strain Graphs

Figure 4 also includes a plot of the maximum plastic equivalent strain in the occupied volume versus the displacement of the ends of the occupied volume. The location of maximum plastic equivalent strain within the model changes throughout the analysis, as local effects influence strain levels.

Initially, plastic strain remains at zero while the load increases linearly, indicating the vehicle is behaving elastically. As the vehicle begins to experience permanent deformation, the maximum plastic strain value begins to grow somewhat slowly. Maximum plastic equivalent strain tends to increase rapidly leading up to localized buckling (first peak) and crippling (second peak).

Because both load and plastic equivalent strain are plotted against the common displacement of the ends of the vehicle, the plastic equivalent strain at a particular load value will occur at a particular displacement read from the graph. The three regions on the graph (elastic, limited plastic deformation, and crippling) correspond to the three Options of the Criteria and Procedures.

\section{CRITERIA}

The regulation governing occupied volume strength for passenger rail equipment can be found at 49 CFR 238.203. This regulation applies to Tier I passenger equipment, operated at speeds no greater than $125 \mathrm{MPH}$. In part, this regulation states:

$\S 238.203$ Static end strength.

(a)(1) Except as further specified in this paragraph or in paragraph (d), on or after November 8, 1999 all passenger equipment shall resist a minimum static end load of 800,000 pounds applied on the line of draft without permanent deformation of the body structure.
For various reasons, an operating authority may wish to place into service equipment that does not comply with all of the FRA Regulations. The waiver process has been put into place as a means of accomplishing this, for vehicles that demonstrate equivalent levels of safety to compliant vehicles.

One area of regulation for which relief is frequently sought is §238.203. In the case of equipment designed to be operated on the general railroad system throughout Europe, a different set of requirements exist than in the United States. Specific to structural strength, the analogous requirement to $\$ 238.203$ is published in EuroNorm (EN) 12663 "Railway Applications Structural Requirements of Railway Vehicle Bodies" [3]. Depending on the classification of the rail vehicle, the body structure must resist a different compressive load value without yielding. The largest load magnitude, corresponding to heavy passenger rail vehicles (Category P-I), is $2000 \mathrm{kN}$ ( 450 kips).

Within the field of passenger equipment design, a relatively recent design development has seen the inclusion of CEM features within more vehicle designs. The CEM methodology does not rely on a uniformly strong passenger car to protect occupants in a collision. Rather, CEM places sacrificial areas within unoccupied regions of the car that are designed to deform and absorb collision energy. However, CEM does not mean OVI may be overlooked. An occupied volume must still be constructed in such a manner as to allow the "crush zones" to deform and absorb energy while still maintaining occupied volume for occupants to ride out the collision.

What CEM does do is to change the load path through the occupied volume during collisions, as opposed to during normal service. In normal railroad operations, the train's buff (compressive) loads are transmitted through the coupling mechanism at the end of the vehicle, into the body structure, and out through the coupling mechanism at the other end of the vehicle. During a collision, the loads may follow a different path. The loads are transmitted through the energy-absorbing devices, which may or may not be in-line with the service load path. Because preservation of occupied volume is essential for occupant survivability in a collision, it is important that the behavior of the occupied volume be understood when loaded in a manner similar to the manner in which it will be loaded during a collision.

An additional set of requirements applicable to equipment operating on the European railroad network exists in EN15227 [4]. This standard includes the requirement that, for CEM equipment, "[t]he structure forming the survival spaces shall remain intact and resist the maximum forces exerted upon it during the full collapse sequence of the energy absorbing elements." EN15227 also includes a scenario-based requirement for preservation of survival space in various train collisions. The scenario is designed to analyze the train-level 
occupied volume performance under various collision conditions. While there is no direct analogue to the scenariobased requirement within U.S. regulations, a scenario requirement has been included with the Criteria and Procedures document [1]. The scenario included in the Criteria and Procedures document is discussed in a companion paper to this one [5].

Three options have been developed by the ETF as alternatives to the stated regulation to demonstrate sufficient OVI in equipment that does not comply with the regulation. A piece of equipment must demonstrate compliance with either the regulation or at least one of the options. As discussed in detail in the Procedures section of this document, compliance may be demonstrated through a combination of testing and analysis.

\section{Option A}

Passenger equipment shall resist a minimum quasi-static end load of 800,000 pounds applied on the collision load path without permanent deformation of the occupied volume.

\section{Option B}

Passenger equipment shall resist a minimum quasi-static end load of 1,000,000 pounds applied on the collision load path with limited permanent deformation of the occupied volume. This load shall be supported without exceeding either of the following two conditions:

- Local plastic strains of 5\%; or

- Vehicle shortening of $1 \%$ over any $15 \mathrm{ft}$ of the occupied volume.

\section{Option C}

Passenger equipment shall resist a minimum quasi-static end load of 1,200,000 pounds applied on the collision load path without crippling the body structure. Crippling of the body structure is defined as the maximum point on the loaddisplacement characteristic.

\section{EXAMPLE PROCEDURES}

This section includes a set of example procedures for alternatively evaluating OVI. Procedures for testing a car body, analyzing the OVI, and validating the analytical model with test data are discussed in further detail in this section. The example procedures outlined here are one way to demonstrate compliance with one of the three options. Other procedures may be used.

For any of the three Options selected for evaluation, it is envisioned that finite element analysis (FEA) will be utilized in demonstrating a particular design's capability of meeting the option. While performing a full-scale test of a vehicle is not disallowed, in Options B and C some permanent deformation is permitted to occur within an occupied volume. This permanent deformation would likely prevent a full-scale test article from being placed into revenue service following the test without significant modification. Thus, a properly-validated FEA can be more cost- and time-effective than destructive testing of a full-scale test article.

\section{Testing Procedures}

It is expected that quasi-static compression testing will be used as part of a program of model validation for any of the three options. The test procedure used should follow a recognized national or international standard. Regardless of the standard employed, the end load should be a minimum of 337,000 lbs. This load may otherwise be applied in accordance with the selected standard. The standard used shall be clearly identified and a copy shall be furnished to FRA if requested.

In all cases, the test procedures should include loading the car to some intermediate load value less than the maximum load to be applied. This allows verification that the instrumentation is functioning properly, as well as provides data that can be used to confirm the predicted behavior at the ultimate test load.

Critical measurements for any test should include strains throughout the structure as well as deformation of the occupied volume over the course of the test.

\section{Analysis Procedures}

Analysis may be used to demonstrate compliance with any of the three options for OVI. Any analysis that is being used to demonstrate a given design's ability to meet the option must be properly validated with test data. Regardless of the option being employed, the procedures for modeling the occupied volume should be the same, aside from the load magnitude being applied.

\section{Geometry}

The entire occupied volume (for both passengers and crewmembers) should be modeled. If the car body is symmetric, symmetry boundary conditions may be employed to facilitate efficient execution. Couplers (both front and rear), articulations, and CEM components may be removed from the model. Mesh size should be sufficiently fine to capture stress details where necessary throughout the model.

\section{Materials}

Materials used in the model should include their elasticplastic stress-strain behavior. Where possible, material properties derived from material test results should be used. Material properties may be assumed to be independent of the rate of deformation. Failure modeling of connections (welds, rivets, bolts, etc.) is not required provided the analysis does not indicate critical stresses/strains in the vicinity of the connectors. 


\section{Boundary Conditions}

Vertical support for the car body model should be provided at the locations where it would be provided in a physical car body. In most cases, this would be at the points where the trucks' secondary suspension elements interface with the car body. If an articulation is used in the design, vertical support may also be provided at the location of the articulation in the physical car structure.

Longitudinal restraint may be provided at the rear end of the car by means of a rigid wall. The wall may be divided into multiple parts to facilitate measurement of reaction forces at the floor level, wall level, and roof level. The rigid wall is not permitted to move in any direction.

Lateral restraint may be accomplished through a longitudinal-vertical symmetry boundary condition. If a longitudinal-vertical symmetry plane is not employed in a model, lateral restraint may be provided through the use of a reasonable coefficient of friction between the rear reaction wall and the car structure.

\section{Loading}

Loading of the occupied volume should resemble the type of loading the occupied volume on a physical car would experience during a collision situation. Loads should be applied along the collision load path based upon the details of the specific design being evaluated. If collision energy absorbers are removed from the model, loading of the structure may take place through the energy absorber supports at the end of the occupied volume. One way of applying these loads is through the use of a rigid plate at each energy absorber support location. Each rigid plate would have a prescribed displacement that increases with time, allowing the plate to contact the energy absorber support in a manner similar to that of the energy absorber reacting against its support.

The prescribed load rate applied to each rigid plate should be the same for a given car design. The rate should be chosen carefully to avoid introducing dynamic effects to the simulation. For all three Options, OVI is to be demonstrated for a quasi-static loading case. It is important to ensure that the model is predicting the quasistatic behavior of the car free from dynamic effects.

An analysis conducted in accordance with either of the below two conditions should be considered quasi-static.

\section{Condition One}

For a given simulated load rate, the load at the live end of the model should be the same as the load at the fixed end. Load at the reaction end may vary by up to $+/-5 \%$ of the load at the live end of the model for the analysis to be considered quasi-static. Figure 5 depicts two example load-displacement characteristics for the same model, one generated at the point of load application and one generated at the reaction location. A +/- 5\% envelope is plotted on this example graph to demonstrate the quasistatic nature of the analysis.

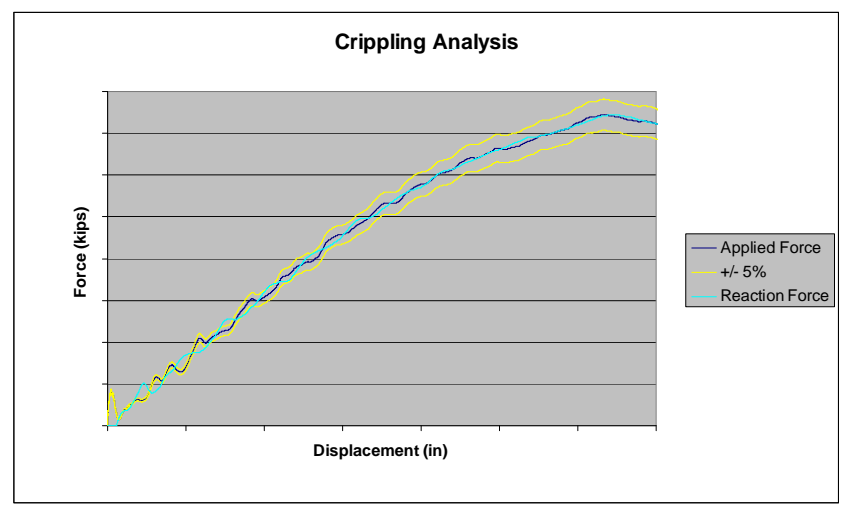

Figure 5. Example Load-displacement Characteristics at Front and Back End with 5\% Envelope

\section{Condition Two}

The ratio of kinetic energy-to-strain energy within the structure should be small $(<5 \%)$. The ratio of kinetic energy-to-strain energy may exceed $5 \%$ during the first $10 \%$ of the total simulation time without invalidating the analysis as quasi-static. Figure 6 shows a sample graph of kinetic energy-to-internal energy plotted against the normalized analysis time.

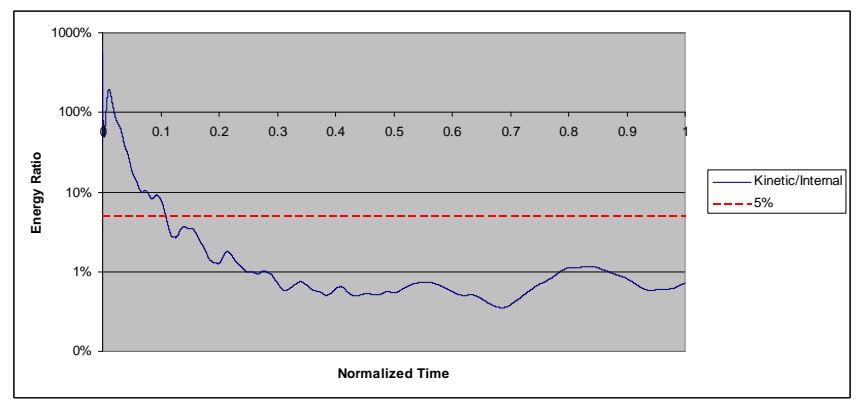

Figure 6. Example Ratio of Kinetic Energy-to-Strain Energy for Analysis

\section{Validation Procedures}

If analysis is used to demonstrate compliance with any of the three options, the model used must be validated with test data. The model should be validated with data from a compressive strength test of the occupied volume. The load may be applied to the vehicle in a manner consistent with the governing design standard, with an end load magnitude no less than $1500 \mathrm{kN}$ (337,000 lbs) regardless of the load magnitude required by the design standard. 
The same occupied volume finite element model should be used to simulate the test and demonstrate compliance with an Option. For validation analyses, the effects of gravity should be included in the model. Critical measurements to be compared between tests and analyses include the overall decrease in length of the car, the vertical deflection of the car, and the strain state in the car. Strain measurement locations should be chosen to minimize the local effects resulting from features such as welds, sharp corners, and geometric features that are not relevant to the occupied volume's global behavior. For displacement data, analytical results within $+/-10 \%$ of the test measurements should be considered as acceptable validation. For strain data, analytical results within $+/-20 \%$ of the test measurements should be considered as acceptable validation.

\section{Procedures Specific to Option A}

Compliance with Option A requires demonstrating (through testing or a combination of testing and analysis) that at a load of 800,000 lbs distributed along points in the collision load path, the occupied volume experiences no permanent deformation.

For a test, the pass/fail criterion of "no permanent deformation" may be verified by a lack of visible damage, as well as displacement measurements indicating no permanent set in the overall dimensions of the car. Additionally, strain gage results should not indicate stresses above the yield stress of the material.

For compliance demonstrated by analysis, highly localized areas of stress exceeding yield ("hotspots") may be allowed as discussed below. An analysis may include the designated energy-absorbing elements, or these may be removed. If the energy-absorbing elements remain within the model, permanent deformation within designated energy-absorbing areas should not constitute failure to meet Option A.

Plastic strains can develop in a model that would not be apparent in a test under the same conditions, in many cases because of assumptions and simplifications made in any finite element model. For example, sharp corners and idealized member-to-member connections may result in artificially high strains in localized regions. In consideration of this, plastic strains may be permitted within an analysis that otherwise demonstrates meeting Option A under all of the following conditions:

- Plastic analysis of the model shows the affected areas to be small with plastic strain not exceeding $1 \%$;

- With removal of the simulated load there is no permanent set in the overall dimension of the occupied volume; and

- The function of the structure is not compromised
Typical results generated from such analysis would include:

- Contour plots indicating maximum strains in model under 800,000-lb load;

- Contour plots indicating deformation (vertical, lateral, and longitudinal) in model under 800,000-lb load;

- Evidence of model validation, including comparison of test and analytical deflections and stresses;

- Load-displacement characteristic, including loading and unloading behavior for the entire structure; and

- Load-displacement characteristics at each load application point.

\section{Procedures Specific to Option B}

Using a validated finite element model, demonstrate that at a load of 1,000,000 pounds in line with the collision load path, the maximum plastic strain experienced by the structure of the car is $\leq 5 \%$. Under this load condition, vehicle shortening may not exceed $1 \%$ for any $15-\mathrm{ft}$. length of occupied volume.

Plastic strains may develop that exceed the $5 \%$ permitted under Option B. In many cases these strains develop because of assumptions and simplifications made in any finite element model. For example, sharp corners and idealized member-tomember connections may result in artificially high strains in localized regions. In consideration of this, plastic strains exceeding $5 \%$ should be permitted within an analysis that otherwise demonstrates meeting Option B under all of the following conditions:

- The maximum plastic strain value is $<10 \%$; and

- Strains exceeding $5 \%$ are not located on any primary longitudinal load carrying component.

Primary longitudinal load carrying component means an identifiable structural element of the car body that is capable of carrying axial and bending loads, such as side sills, and carries a significant portion of the applied longitudinal load when the load is applied in-line with the collision load path of the car body.

Typical results generated from such analysis would include:

- Contour plots indicating maximum strains in the model under the 1,000,000-lb load;

- Contour plots indicating deformation (vertical, lateral, and longitudinal) in the model under the 1,000,000-lb load;

- Evidence of model validation, including comparison of test and analytical deflections and stresses;

- Load-displacement characteristic up to 1,000,000 lbs for the entire structure; and

- Load-displacement characteristics at each load application point. 


\section{Procedures Specific to Option C}

Using a validated finite element analysis, demonstrate that the crippling load is greater than $1,200,000$ lbs. This property can be readily observed on a load-displacement characteristic. Note that for vehicles with crippling loads exceeding 1,200,000 lbs, only that portion of the characteristic showing no crippling at $1,200,000$ lbs needs to be provided. Figure 7 shows a sample load-displacement characteristic taken from the front and back of the same car model. This example characteristic only shows the force up to $1,200,000 \mathrm{lbs}$, at which point crippling has not yet occurred.

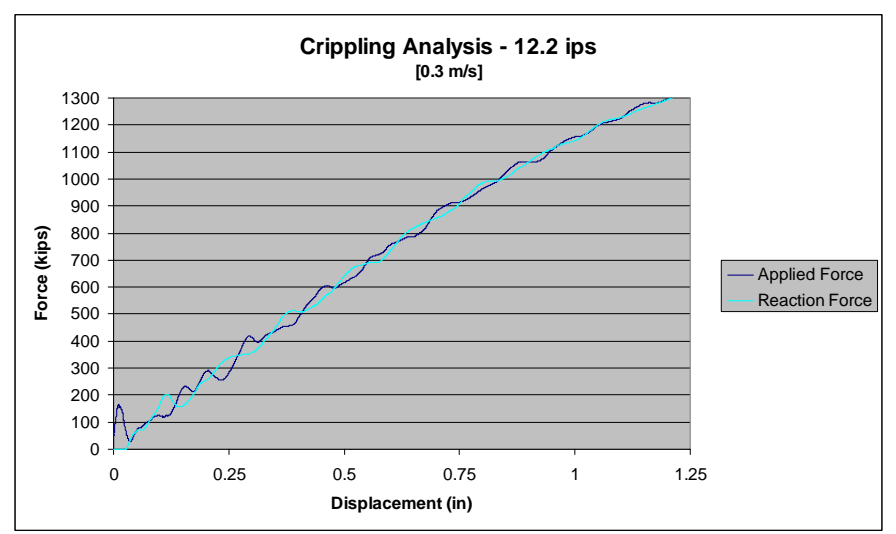

Figure 7. Example Load-displacement Characteristic Showing Crippling Load Exceeds 1.2 Million lbs.

Typical results generated from such analysis would include:

- Contour plots indicating maximum strains in the model under the 1,200,000-lb load;

- Contour plots indicating deformation (vertical, lateral, and longitudinal) in the model under the 1,200,000-lb load;

- Evidence of model validation, including comparison of test and analytical deflections and stresses;

- Load-displacement characteristic up to $1,200,000 \mathrm{lbs}$ for the entire structure; and

- Load-displacement characteristics at each load application point.

If analysis is taken beyond crippling, contour plots for strain and deflection at crippling load should also be provided.

\section{CONCLUSIONS}

An RSAC Engineering Task Force has been working to develop Criteria and Procedures for evaluating the crashworthiness of alternatively-designed passenger equipment. Because these vehicles are not designed to the applicable U.S. regulations, a methodology was sought to compare the performance of these vehicles to the performance of those that are designed to be compliant with the regulations. Criteria and Procedures have been developed in a number of areas of crashworthiness, including OVI.
Three Options have been developed to evaluate the OVI of passenger rail vehicles that are not compliant with the existing domestic regulation. The Options were developed to provide some variability in evaluating vehicles that may be designed with different methodologies in mind.

Option A's Criteria are an 800,000 pound quasi-static compressive load applied along the collision load path with the occupied volume experiencing no permanent deformation.

Option B's Criteria are a $1,000,000$ pound quasi-static compressive load applied along the collision load path with only a limited amount of allowable permanent deformation.

Option C's Criteria are a 1,200,000 pound quasi-static compressive load applied along the collision load path without crippling the occupied volume.

Example procedures have also been developed as an aid to applying the Criteria. Example procedures are included for performing a non-destructive compression test of a car body, conducting a quasi-static finite element analysis of a compressive strength test, and validating the FE model with test data.

\section{ACKNOWLEDGEMENTS}

The Volpe Center's contribution to ETF was part of the Federal Railroad Administration's Equipment Safety Research Program under the Office of Research and Development. The ETF was led by the Office of Safety of the Federal Railroad Administration. Volpe participated in the ETF group as technical advisors and coordinated the development of drafting the ETF report. Some of the results contributed to the example procedures and key results were provided by industry participating in the ETF. At the time of this paper's publication, the ETF has not finalized its recommended Criteria and Procedures.

Jo Strang, FRA Associate Administrator of Railroad Safety, and Robert C. Lauby, FRA Deputy Associate Administrator for Regulatory and Legislative Operations, led the Engineering Task Force. Cindy Gross and Larry Wolverton, FRA, coordinated and managed the task force. Martin Schroeder of APTA coordinated the APTA effort. Dan Alpert, FRA Trial Attorney, provided review and counsel.

The research described in this paper was performed as part of the Equipment Safety Research Program sponsored by the Office of Research and Development of FRA. Eloy Martinez, Program Manager, Office of Railroad Policy and Development, managed the research-related effort. Kevin Kesler is Chief of the Equipment and Operating Practices Division. Grady Cothen, former Deputy Associate Administrator for Safety 
Standards and Program Development, established the ETF and has coordinated FRA's regulations development and the passenger rail equipment crashworthiness research since the inception of this research.

\section{REFERENCES}

1. "Technical Criteria and Procedures for Evaluating the Crashworthiness and Occupant Protection Performance of Alternatively-Designed Passenger Rail Equipment for Use in Tier I Service,” 2010. [PENDING]

2. Carolan, M., "Evaluation of Occupant Volume Strength in Conventional Passenger Railroad Equipment" Tufts University Master's Thesis, May 2008

3. "Railway Applications - Structural Requirements of Railway Vehicle Bodies.” EN12663:2000.

4. "Railway Applications - Crashworthiness Requirements for Railway Vehicle Bodies.” prEN 15227:2007.

5. Jacobsen, K., Llana, P., Tyrell, D.C. "Collision Scenarios for Assessing Crashworthiness of Passenger Equipment." American Society of Mechanical Engineers, Paper No. RTDF2010-42033, October 2010. 